\title{
PERFORATED PEPTIC ULCER: A CLINICAL ANALYSIS AND OUTCOME
}

\author{
Bijit Gogoi ${ }^{1}$ \\ ${ }^{1}$ Assistant Professor, Department of Surgery, Gauhati Medical College.
}

\section{ABSTRACT}

\section{BACKGROUND}

The incidence of perforated peptic ulcer is approximately 7-10 cases per one lakh population per year. Perforation is seen in about $7 \%$ of patients hospitalized for peptic ulcer disease. Peptic ulcer perforation, which can be gastric/duodenal perforation can be a serious life-threatening condition if not detected early and treated urgently. Peptic ulcer disease has decreased considerably worldwide with the advent of potent anti-ulcer medicines, but its complication like peptic ulcer perforation has not. Our study is to analyse the clinical, radiological and management related findings in influencing the outcome of patients of peptic ulcer perforation after surgery.

\section{MATERIALS AND METHODS}

A series of 47 patients of peptic ulcer perforation were evaluated. Patients expiring within six hours of admission were not included in this study.

\section{RESULTS}

Age of the patients ranged from 17-80 years. The incidence of perforation was highest in the age group of 41-50 years (31.9\%). Out of 47 patients, $41(87.2 \%)$ survived.

\section{CONCLUSION}

The incidence of perforation was highest in the age group of 41-50 years. Prognosis becomes poor with age, delayed treatment, shock at admission and concomitant diseases. Direct repair of the perforation with pedicled omentum gave excellent results.

\section{KEYWORDS}

Peptic Ulcer Perforation, Peritonitis, Graham's Pedicled Omental Patch, Outcome.

HOW TO CITE THIS ARTICLE: Gogoi B. Perforated peptic ulcer: a clinical analysis and outcome. J. Evolution Med. Dent. Sci. 2016;5(22):1195-1198, DOI: 10.14260/jemds/2016/277

\section{INTRODUCTION}

The incidence of perforated peptic ulcer is approximately 7-10 cases per one lakh population per year. Perforation is seen in about $7 \%$ of patients hospitalized for peptic ulcer disease. It is the first manifestation of the disease in about $2 \%$ of patients with duodenal ulcer. In the duodenum, the ulcers that perforate are located anteriorly and the saying that anterior ulcers perforate and posterior ulcers bleed still holds good. In contrast, gastric ulcers may perforate freely through either the anterior or posterior wall. Peptic ulcer perforation, which can be gastric/duodenal perforation is a serious life-threatening condition. It has to be detected early and treated immediately if the patient is to survive. In short, it is a surgical emergency. Peptic ulcer perforation patients usually come with symptoms and signs of peritonitis followed by septicaemia. Even postoperatively, morbidity can be as high as $20-50 \%$ and mortality ranging from $3-40 \%$. The worldwide prevalence of peptic ulcer disease has decreased considerably with the advent of potent antiulcer medicines, but the complications of peptic ulcer like perforation has not followed a similar decrease.

Financial or Other, Competing Interest: None.

Submission 19-02-2016, Peer Review 02-03-2016,

Acceptance 05-03-2016, Published 17-03-2016.

Corresponding Author:

Dr. Bijit Gogoi,

H. No-7, Basisthapur Lane 4,

Wireless, Beltola,

Guwahati-781028.

E-mail: bgogoi123@yahoo.co.in

DOI: $10.14260 /$ jemds $/ 2016 / 277$
Nowadays besides laparotomy, for repair of the perforation, laparoscopic repair of the perforation is also being done in well-equipped centers and by experienced laparoscopic surgeons well versed in the art of suturing laparoscopically with good results. Our study is to analyse the clinical, radiological and management related findings in influencing the outcome of patients with peptic ulcer perforation after surgery.

\section{MATERIALS AND METHODS}

A series of 47 consecutive patients of peptic ulcer perforation admitted and treated at Gauhati Medical College and Hospital during the period from January 2012 to December 2012 were evaluated. All the patients suffered from peptic ulcer perforation on laparotomy. Patients expiring within six hours of coming to the hospital were not included in the study. The clinical profile of the patients were duly recorded which included age, sex, type of perforation, associated medical illness, shock and any other associated features.

The patients who presented in the emergency department had an immediate chest X-ray (Erect), plain picture abdomen (Erect) and ultrasound abdomen done and cxr findings like gas under right dome of diaphragm, USG finding like free fluid and free air in the peritoneal cavity were noted. Patients were resuscitated and put up for surgery depending on their clinical condition. Patients who were in shock were properly resuscitated before surgery with IV crystalloids and colloids as needed.

Emergency exploratory laparotomy with repair of the perforation with Graham's patch with thorough normal saline 
wash of the peritoneal cavity was done in the operated patients. The patients received IV fluids, IV antibiotics, proton pump inhibitors and multivitamins. All complications were noted and treated accordingly. Patient's outcome was noted at the time of discharge and at 3 weeks, 3 months and 6 months followup. All records were then closely scrutinized and the clinical findings, radiological findings and the management were analysed with the outcome. During followup of the patients, the patient's symptoms if any were enquired and graded according to the modified Visick classification.

\section{Grade}

1. No symptoms, excellent results.

2. Mild symptoms, good results.

3. Moderate symptoms, easily controlled by medications.

4. Severe symptoms, requiring constant medication or reoperation.

\section{RESULTS}

The study consisted of 47 consecutive patients of peptic ulcer perforation. All 47 patients had emergency exploratory laparotomy for the peptic ulcer perforation. Age of the patients ranged from 17 to 80 years. The incidence of perforation was highest in the age group of $41-50$ years (31.9\%). Mean age of the patients in our series was 42.9 years. Mean age of survivors was 42.2 years and 45.3 years in those who expired. There were 43 males (91.5\%) out of which $39(83 \%)$ survived and 4 females $(8.5 \%)$ out of which $2(4.3 \%)$ survived and this difference was statistically insignificant ( $>00.05)$. The male/female ratio was 10.8:1. Two patients were in shock at the time of admission. The highest incidence of perforation was seen in the month of January. Most of the patients belonged to the lower socioeconomic group and were illiterate or semiliterate.

The perforation usually occurred in the morning or afternoon; 9 (19.1\%) patients presented within 24 hours of the occurrence of symptoms, $29(61.7 \%)$ patients within 24 48 hours and $9(19.1 \%)$ patients after 48 hours. The patients presented with symptoms like sudden severe upper abdominal pain in all $47(100 \%)$ patients, abdominal distension in all $47(100 \%)$ patients and nausea in $20(42.5 \%)$ and vomiting in $18(38.3 \%)$ patients. Abdominal tenderness was seen in all $47(100 \%)$ patients and clinical signs of peritonitis like abdominal rigidity in all 47 (100\%) patients. The patients came with symptoms, which occurred 1-4 days back; $30(63.8 \%)$ patients gave previous history of symptoms relating to peptic ulcer disease and their previous symptoms ranged from 6 months to 10 years and none of them were on regular antiulcer medication.

$2(4.2 \%)$ patients gave history of taking NSAID (Nonsteroidal anti-inflammatory drugs) before the incident; 5 patients $(10.6 \%)$ gave history of alcohol consumption and 38 $(80.85 \%)$ patients gave history of smoking/tobacco use. At the time of surgery $3(6.3 \%)$ patients had gastric ulcer perforation, $4(8.5 \%)$ patients had prepyloric perforation and the rest 40 (85.1\%) had duodenal ulcer perforation. On biopsy 1 (2.1\%) of the gastric ulcer perforations turned out to be adenocarcinoma. All 47 (100\%) patients had plain picture abdomen and chest X-ray done and free gas under right diaphragm was seen in $46(95.7 \%)$ patients.

They also had urgent ultrasonography abdomen done, which demonstrated free air foci and fluid collection in the peritoneal cavity in $46(95.7 \%)$ patients. Abdominal paracentesis revealed turbid fluid in 46 (95.7\%) patients; 2 patients came with raised serum creatinine levels and 5 patients with associated bilateral pleural effusion on USG. Sealed perforation was seen in 1 patient during laparotomy. During surgery in all the patients, the abdomen was opened via a midline incision. There was escape of gas on opening the abdomen in most of the cases. Associated fluid collection was found in the peritoneal cavity in all the patients. The collected fluid amount ranged from $250 \mathrm{~mL}$ to $1-2$ litres.

In all the patients, the fluid was a mixture of bile and gastric juice and associated seropurulent fluid was seen in 25 $(53.2 \%)$ patients. In a few patients undigested food particles like rice and vegetables were seen coming out of the perforation site, particularly when the size of the perforation was bigger. Fibrinous flakes were usually present near the perforation and over the loops of intestine. The size of the perforations ranged from $4 \mathrm{~mm}$ to $8 \mathrm{~mm}$. In operative treatment, all the patients had Graham's repair of the perforation with a pedicled greater omentum applied directly over the perforation and sutured with 2-0 vicryl and a thorough normal saline wash of the entire peritoneal cavity done with suction of the fluid; 2 drains were placed on either flank for the fluid to come out and to observe for any postoperative leak from the perforation site and the drains were removed usually on day five.

One patient had a retrocolic gastrojejunostomy in addition to the repair, because of gastric outlet obstruction with dilated stomach. In the outcome, postoperative complications were seen in 30 patients. The complications were surgical site infection in 25 patients, pulmonary complications with tachypnoea, crepitations and rhonchi in five patients, fever in two and excess serous discharge from drain site in two patients. The duration of hospital stay ranged from 9 days to 22 days. The patients with complications had to stay for a prolonged period ( $\geq 10$ days) and this was statistically insignificant $(\mathrm{p}>0.05)$. In our study, six patients died with a mortality rate of $12.8 \%$. In the followup, at each visit the patient's symptoms if any were reviewed and classified according to the Visick grading system. Visick grade I: $41(87.2 \%)$ patients, Visick grade II: $4(8.5 \%)$ patients, Visick grade III: $2(4.3 \%)$ patients and Visick grade IV: nil patients.

\section{DISCUSSION}

In our series, peptic ulcer perforation was highest in the age group of 41-50 years. The youngest patient was 17 years and the oldest was 80 years of age. Peptic ulcer perforation affected more males than females with a male:female ratio of 10.8:1 in our series. Chalya and Mabula et al. (2011). ${ }^{1}$ found perforated peptic ulcer to be very common in the fourth decade of life. They have reasoned it to be the increased alcohol intake amongst the males, because alcohol causes damage to the gastric mucosa and increases acid secretion. A study by AK Dev and S Paul et al. (1994). ${ }^{2}$ showed the peak incidence of perforation of duodenal ulcer in the age group of 46-55 years. Plummer et al. (2004). ${ }^{3}$ in his series of 97 cases found mean age of male patients to be 49 years, ranging from 21 to 85 years. The mean age of female patients was 74 years ranging from 50 to 93 years.

Chalya and Mabula et al. (2011). ${ }^{1}$ found the perforation to affect more males than females with a male:female ratio of $1.3: 1$. In the western world earlier the male:female ratio was $2: 1$, but now perforations mostly occur in the elderly female 
patients and NSAIDs were usually responsible for these perforations, reported John Primrose (2004).4 A study by S Vijaya M Rao et al. (2014). ${ }^{5}$ found the perforation to be more common in men deducing it to be on the basis of greater hardship, alcoholism and NSAIDs. Helicobacter pylori infection is responsible for more than $90 \%$ of duodenal ulcers and up to $80 \%$ of gastric ulcers, Sivri (2004). ${ }^{6}$ Ahmed (2005). ${ }^{7}$ In recent times helicobacter pylori infection and NSAIDs are said to be the two main causes of peptic ulcer, Sivri (2004). ${ }^{6}$ Helicobacter pylori test was not done in our study because of lack of test materials. In our series, highest incidence of perforation was seen in the month of January. S Vijaya Rao et al. (2014). ${ }^{5}$ showed high incidence of perforation in the winter season. In our series, $30(63.8 \%)$ patients gave previous history of peptic ulcer disease.

Chalya and Mabula et al. (2011). ${ }^{1}$ reported that more than sixty percent of their patients did not give history of peptic ulcer disease. S Vijaya M Rao et al. (2014). ${ }^{5}$ in their series showed that $68 \%$ of their patients gave symptoms of dyspepsia/peptic ulcer before perforation. Nuhu et al. (2009). ${ }^{8}$ in Nigeria said that patients with history of peptic ulcer disease was $71 \%$ in their series. In our study, most of the patients belonged to the low income and illiterate/semiliterate group and similar was the scenario in the reports of S Vijaya M Rao et al. (2014). ${ }^{5}$ Chalya and Mabula et al. (2011). ${ }^{1}$ and Nuhu et al. (2009).8 This meant that these patients were less aware of their disease and their sequelae and so also had less access to proper health care. Most of the patients in our series reported after 24 hours to the emergency department as was also the finding with Chalya and Mabula et al. (2011). ${ }^{1}$ S Vijaya Rao et al. (2014). ${ }^{5}$ Nuhu et al. (2009). ${ }^{8}$

Mortality and morbidity of the patient increases with delayed reporting to the hospital, because by that time toxicity sets in with pulmonary complications and renal compromise which becomes very difficult to correct. Delay of more than 24 hours increased lethality seven to eight fold, the complication rate threefold and the length of hospital stay twofold in a study from Norway, Svanes, Lie et al. (1994). ${ }^{9}$ The diagnosis of perforation of peptic ulcer was made from the clinical features of the patient, presence of gas under right diaphragm on chest $\mathrm{X}$-ray and plain picture abdomen, abdominal USG showing air and free fluid and finally during the time of surgery. Chalya et al. (2011). ${ }^{1}$ could detect free air on chest X-ray/plain picture abdomen in $65.8 \%$ cases. In our series 46 (95.7\%) patients showed gas under right diaphragm.

The absence of gas under diaphragm in some cases could be because the radiograph was taken in a lying down posture. Chalya et al. (2011). ${ }^{1}$ reported that duodenal ulcer perforation was the common perforation in their series and their duodenal to gastric ulcer ratio was 12.7:1. Svanes et al. (2000)..$^{10}$ in his series of 1483 patients of peptic ulcer perforation had a ratio of 9:1. Our series showed a duodenal/gastric ulcer perforation ratio of 5.7:1. Chalya et al. (2011). ${ }^{1}$ in his study preferred Graham's omental patch for closing the perforation with either a pedicled omental graft or a free graft of omentum. In our study, we did Graham's pedicled omental repair directly applied over the perforation in all our patients with excellent results.

S Vijaya Rao et al. (2014). ${ }^{5}$ in their series did simple closure of the perforation with omental graft reinforcement with very good results. Definitive surgery like gastric resections and truncal vagotomy for reducing acid secretion has become obsolete with the availability of proton pump inhibitors and so the actual operative treatment for perforated peptic ulcer is repair of the perforation with omental patch, Varcus et al. (2013).11 Sneider et al. (2010).12 Bertleff et al. (2010). ${ }^{13}$ The operative management has now changed to minimal access laparoscopic surgery in select patients with reduced postoperative pain, wound infection and burst abdomen in centers where laparoscopic emergency surgery facilities are available with requisite laparoscopic suturing expertise Thorsen, Glomsaker et al. (2011). ${ }^{14}$ Varcus et al. (2013). ${ }^{11}$ Sneider et al. (2010). ${ }^{12}$ Bertleff et al. (2010).13

Postoperative complications in our series were commonly pneumonia and wound infections with excess serous discharge in two patients via drain, which was corrected with albumin infusion. All six patients (12.8\%) who expired had associated pulmonary complications. Chalya et al. (2011). ${ }^{1}$ reported surgical site infection as their commonest complication and added that complications could be somewhat lessened by good antibiotics and good preoperative resuscitation and to which we add good postoperative care and proper surgery. Bertleff et al. (2010).13 reported pneumonia and wound infection as the commonest complication and death at 5-11\% in literature reviews, which was seen in our series too.

S Vijaya Rao (2014). 5 reported pulmonary complications at $15 \%$ and wound infection at $16 \%$ in their series. Imhof et al. (2008). ${ }^{15}$ said that mortality after surgery for peptic ulcer perforation ranges from 6-10\%. Zittel et al. (2000). ${ }^{16}$ Sarosi et al. (2005). ${ }^{17}$ say that four factors can increase the mortality rate even by $100 \%$ and these are aged $>60$ years, delayed treatment ( $>24 \mathrm{hrs}$.), shock at admission (Systolic BP $<100$ $\mathrm{mmHg}$ ) and concomitant diseases. Zittel et al. (2000).16 Harbison et al. (2005). ${ }^{18}$ reported that gastric ulcers have a two to threefold increased mortality risk. Boey's score is a good test for predicting outcome in perforated patients. The Boey's score depends on factors like shock on admission, confounding medical illness and prolonged perforation, Bertleff et al. (2010).14 Boey, Wong et al. (1982). ${ }^{19}$ In the followup of the patients, Visick grading system was used to assess the patients (2011). ${ }^{1}$

\section{CONCLUSION}

1. 47 cases of peptic ulcer perforation were studied; 2 . The incidence of perforation was highest in the age group of 41-50 years; 3. Prognosis becomes poor with age, delayed treatment, shock at admission and concomitant diseases; 4. Incidence of perforation was highest in winter; 5 . Most of the patients belonged to the lower socioeconomic group; 6. NSAID use increases the risk of perforation besides predisposition to alcohol and smoking; 7. Peptic ulcer perforation can be a symptom of gastric cancer as seen in one of our patients; 8 . Postoperative complications were commonly pneumonia and wound infection; 9. Direct repair of the perforation with pedicled omentum gave excellent results.

\section{REFERENCES}

1. Phillipo L Chalya, Joseph B Mabula, Mheta Koy, et al. Clinical profile and outcome of surgical treatment of perforated peptic ulcers in northwestern Tanzania: a tertiary hospital experience. World J Emerg Surg 2011;6:31. 
2. Dev AK, Paul S, Bhattarcharjee N, et al. Perforated duodenal ulcer. Ind J Surg 1994;56:222-27.

3. Plummer JM, Mcfarlane MEC, Newnham MS. Surgical management of perforated duodenal ulcer. The changing scene. West Indian Med J 2004;53(6):378-81.

4. John Primrose, Bailey and Love's short practice of surgery 2004;24th edition:pp 1045.

5. Vijaya S, Rao M, Siddharth O. A clinical study of duodenal ulcer perforation. Ind Jour of App research 2014;pp 23036.

6. Sivri B. Trends in peptic ulcer pharmacotherapy. Fundam Clin Pharmacol 2004;18(1):23-31.

7. Ahmed N. 23 years of the discovery of helicobacter pylori: is the debate over? Ann Clin Microbiol Antimicrobiol 2005;4:17.

8. Nuhu A, Madziga AG, Gali BM. Acute perforated duodenal ulcer in Maiduguri. The Internet Journal of Surgery 2009;6:1.

9. Svanes C, Lie RT, Svanes K, et al. Adverse effects of delayed treatment for perforated peptic ulcer. Ann Surg 1994;220:168-75.

10. Svanes C. Trends in perforated peptic peptic ulcer: incidence, aetiology, treatment and prognosis. World J Surg 2000;24:277-283.

11. Varcus F, Lazar F, Beuran M, et al. Laparoscopic treatment of perforated duodenal ulcer-a multicenter study. Chirurgia Bucur 2013;108:172-176.
12. Sneider EB, Cahan MA, Litwin DE. Laparoscopic repair of acute surgical diseases in the $21^{\text {st }}$ century. Minerva Chir 2010;65:275-296.

13. Bertleff MJ, Lange JF. Laparoscopic correction of perforated peptic ulcer: first choice? A review of literature. Surg Endosc 2010;24:1231-1239.

14. Thorsen K, Glomsaker TB, von Meer A, et al. Trends in diagnosis and surgical management of patients with perforated peptic ulcer. J Gastrointest Surg 2011;15(8):1329-1335.

15. Imhof M, Epstein S, Ohmann C, et al. Duration of survival after peptic ulcer perforation. World J Surg 2008;32:408412.

16. Zittel TT, Jehle EC, Becker HD. Surgical management of peptic ulcer disease today: indication, technique and outcome. Langenbecks Arch Surg 2000;385:84-96.

17. Sarosi Jr GA, Jaiswal KR, Nwariaku FE, et al. Surgical therapy of peptic ulcers in the $21^{\text {st }}$ century: more common than you think. Am J Surg 2005; 190:775-779.

18. Harbison SP, Dempsey DT. Peptic ulcer disease. Curr Prob Surg 2005;42:346-454.

19. Boey J, Wong J, Ong GB. A prospective study of operative risk factors in perforated duodenal ulcers. Ann Surg 1982;195(3):265-269. 